MATHEMATICS OF COMPUTATION

Volume 73, Number 247, Pages 1477-1498

S $0025-5718(03) 01582-5$

Article electronically published on July 7, 2003

\title{
COMPUTING IN GROUPS OF LIE TYPE
}

\author{
ARJEH M. COHEN, SCOTT H. MURRAY, AND D. E. TAYLOR
}

\begin{abstract}
We describe two methods for computing with the elements of untwisted groups of Lie type: using the Steinberg presentation and using highest weight representations. We give algorithms for element arithmetic within the Steinberg presentation. Conversion between this presentation and linear representations is achieved using a new generalisation of row and column reduction.
\end{abstract}

\section{INTRODUCTION}

The groups of Lie type are among the most important structures in modern mathematics. Examples of such groups include reductive Lie groups, reductive algebraic groups, and finite groups of Lie type (which include most of the finite simple groups). Many problems in the representation theory of groups of Lie type have been solved using computers 31], [15. In this paper, we give methods for computing within the groups themselves, a problem which has only recently been tackled [17, [24]. We have implemented these algorithms in the Magma computer algebra system [2. We confine our attention to the untwisted groups; automorphisms and twisted groups will be dealt with in subsequent work.

Computational group theory has three main strands: permutation groups, matrix groups, and finitely presented groups. The permutation group approach has traditionally been the most effective because of the very efficient Schreier-Sims family of algorithms [5]. Recently, effective algorithms for matrix groups over finite fields have started to appear, in particular, the matrix group recognition project based on Aschbacher's theorem [21]. The Todd-Coxeter and Knuth-Bendix algorithms for finitely presented groups [26] are the oldest methods in computational group theory.

Permutation group methods are clearly useless for infinite groups of Lie type. Even for finite groups of Lie type, these methods soon become impractical; for example, the smallest permutation representation of $E_{8}(2)$ has degree

$$
293,091,386,578,365,375 \approx 2.9 \times 10^{17},
$$

well beyond the reach of existing computers [23].

Received by the editor January 24, 2002 and, in revised form, December 15, 2002.

2000 Mathematics Subject Classification. Primary 20G15, 20C40.

Key words and phrases. Group of Lie type, reductive algebraic group, computational algebra.

This paper was written during a stay of the first two authors at the University of Sydney. They wish to thank the institute for its hospitality. 
On the other hand, for any field $\mathbb{F}$, there is a faithful linear representation of $E_{8}(\mathbb{F})$ over $\mathbb{F}$ of degree 248 , which makes it feasible to do element operations (provided we can compute in $\mathbb{F}$ ). Howlett et al. [18] construct matrix representations for finite Chevalley groups; this has been implemented in Magma.

The groundbreaking work of Steinberg 28, [30] gives a presentation for the groups of Lie type. In the presentation for $E_{8}(\mathbb{F})$, each element can be expressed as a word of length at most 368 . This does not necessarily give a finitely presented group, however, because some of our generators are parametrised by the field (more precisely, 240 terms are parametrised by the additive group $\mathbb{F}, 8$ are parametrised by the multiplicative group $\mathbb{F}^{\times}$, and 120 are field independent). A word in this presentation requires less memory than a matrix representation, except for type $A_{n}$ where the memory usage is asymptotically the same. An additional advantage is that there is a normal form for elements (the Bruhat decomposition) which reflects the Lie theoretic structure of the group, thus facilitating the use of Lie theoretic techniques. Another computationally important presentation for finite groups of Lie type is described in [3], [19].

In this paper, we describe methods for doing arithmetic in the Steinberg presentation over any field $\mathbb{F}$ in which computation is possible. We note that Riebeek 24, mainly for $\mathbb{F}=\mathbb{F}_{2}$, and Haller [17], mainly for unipotent subgroups, have done part of this work before. We also give algorithms for conversion between the Steinberg presentation and highest weight representations over the field $\mathbb{F}$. Converting a word to a matrix builds on the methods of de Graaf [1] for Lie algebras. Converting a matrix to a word requires a generalisation of row and column reduction - this is the main result of this paper. Given these conversion algorithms, we can compute with either the presentation or a linear representation, whichever is most effective for the problem at hand.

A motivating application for this work is the matrix group recognition project [21], which provides a framework for decomposing a matrix group over a finite field into a subnormal subgroup chain whose quotients are almost simple. Our methods are useful for this project since most almost simple groups are finite groups of Lie type. First, the Steinberg presentation gives a computer representation of these groups in which conjugacy classes and other such properties can be computed efficiently using Lie theory. Second, our generalised row and column reduction algorithm gives a membership test for these groups as matrix groups, which is necessary for recognising simple groups of Lie type [20].

We use standard group theoretic notation [1]. Our group actions are on the right, so conjugation is given by $x^{y}=y^{-1} x y$ and the commutator is given by $[x, y]=x^{-1} y^{-1} x y$. We occasionally use left conjugation: ${ }^{y} x=y x y^{-1}$.

In Sections 2 and 3, we discuss the computation of root systems and structure constants. Section 4 describes the Steinberg presentation. Section 5 contains element arithmetic and canonical form algorithms for the presentation; an implementation of these algorithms is available in release 2.8 of Magma. We describe algorithms for converting between the Steinberg presentation and highest weight representations in Sections [6 and 7; these will be available in the next release of Magma. In Section 8, we discuss applications of our algorithms and give an example. 


\section{Root DATA}

A root system is the basic structure used for the classification of complex semisimple Lie algebras; a pair of root systems in duality is the structure used for the classification of reductive algebraic groups - such a pair is called a root datum. We assume that all permutation and matrix actions are on the right. This is in keeping with the conventions of computational group theory but leads to differences between our formulas and those found in standard references such as [9], 7], and [27.

2.1. Definition. Let $X$ and $Y$ be free $\mathbb{Z}$-modules of rank $d$ with a bilinear pairing $\langle\circ, \circ\rangle: X \times Y \rightarrow \mathbb{Z}$ putting them in duality. Assume we have a basis $e_{1}, \ldots, e_{d}$ for $X$ and a dual basis $f_{1}, \ldots, f_{d}$ for $Y$, so that $\left\langle e_{i}, f_{j}\right\rangle=\delta_{i j}$. Let $\Phi$ be a finite subset of $X$ and suppose that for each $\alpha$ in $\Phi$ we have a corresponding $\alpha^{\star}$ in $Y$; set $\Phi^{\star}=\left\{\alpha^{\star} \mid \alpha \in \Phi\right\}$ and $\alpha^{\star \star}=\alpha$. We call the elements of $\Phi$ roots and the elements of $\Phi^{\star}$ coroots.

Given a root $\alpha$, we define linear maps $s_{\alpha}: X \rightarrow X$ by $x s_{\alpha}=x-\left\langle x, \alpha^{\star}\right\rangle \alpha$ and $s_{\alpha}^{\star}: Y \rightarrow Y$ by $y s_{\alpha}^{\star}=y-\langle\alpha, y\rangle \alpha^{\star}$. These maps are reflections if one of the following equivalent properties hold: $\left\langle\alpha, \alpha^{\star}\right\rangle=2 ; s_{\alpha}^{2}=1 ;\left\langle x s_{\alpha}, y s_{\alpha}^{\star}\right\rangle=\langle x, y\rangle$ for all $x \in X$ and $y \in Y ; \alpha s_{\alpha}=-\alpha$.

We say that $\mathcal{R}=\left(X, \Phi, Y, \Phi^{\star}\right)$ is a root datum if the following are satisfied for every root $\alpha$ in $\Phi: s_{\alpha}$ and $s_{\alpha}^{\star}$ are reflections; $\Phi$ is closed under the action of $s_{\alpha}$; and $\Phi^{\star}$ is closed under the action of $s_{\alpha}^{\star}$. Furthermore, we require that all our root data be reduced, i.e. if $\alpha$ and $\beta$ are roots with $\beta$ a multiple of $\alpha$, then $\beta= \pm \alpha$. The Weyl group $W$ is the group generated by the reflections $s_{\alpha}$.

2.2. Simple roots and positive roots. The roots $\alpha_{1}, \ldots, \alpha_{n}$ are simple roots for $\mathcal{R}$ if they are a basis of $\mathbb{Q} \Phi \leq \mathbb{Q} \otimes X$ and $\Phi=\Phi^{+} \cup \Phi^{-}$, where $\Phi^{+}$is the set of roots that are linear combinations of the simple roots with nonnegative coefficients and $\Phi^{-}=-\Phi^{+}$. The elements of $\Phi^{+}$are called positive roots and the elements of $\Phi^{-}$ are called negative roots. The coroots corresponding to the simple (resp. positive, negative) roots are called simple (resp. positive, negative) coroots.

We call $n$ the rank and $d$ the dimension of $\mathcal{R}$; if $n=d$, then $\mathcal{R}$ is semisimple. The Cartan matrix of $\mathcal{R}$ is $C=\left[\left\langle\alpha_{i}, \alpha_{j}^{\star}\right\rangle\right]_{i, j=1}^{n}$. Given a root $\alpha=\sum_{i=1}^{n} a_{i} \alpha_{i}$, we define its height by $h(\alpha)=\sum_{i=1}^{n} a_{i}$. We can define a reflection invariant bilinear form on $\mathbb{Q} \otimes X$ by

$$
(x, y)=\sum_{\alpha \in \Phi}\left\langle x, \alpha^{\star}\right\rangle\left\langle y, \alpha^{\star}\right\rangle
$$

and a corresponding norm $\|x\|^{2}=(x, x)$. The fundamental weights $\omega_{1}, \ldots, \omega_{n}$ are the elements of $\mathbb{Q} \Phi$ such that $\left\langle\omega_{i}, \alpha_{j}^{\star}\right\rangle=\delta_{i j}$. An element $\lambda$ of $\mathbb{Q} \otimes X$ is dominant if $\left\langle\lambda, \alpha_{i}^{\star}\right\rangle \geq 0$ for all $i$.

Given an element $w$ in the Weyl group $W$, define

$$
\Phi_{w}=\left\{\alpha \in \Phi^{+} \mid \alpha w^{-1} \in \Phi^{-}\right\}=\Phi^{+} \cap \Phi^{-} w .
$$

The length $l(w)$ is the cardinality of $\Phi_{w} ; W$ contains a unique longest element, which we denote $w_{0}$.

\section{Lemma 2.1.}

(1) Given a reduced expression $w=s_{\beta_{1}} \cdots s_{\beta_{l}}$, we have

$$
\Phi_{w}=\left\{\beta_{l}, \beta_{l-1} s_{\beta_{l}}, \beta_{l-2} s_{\beta_{l}-1} s_{\beta_{l}}, \ldots, \beta_{1} s_{\beta_{2}} \cdots s_{\beta_{l}}\right\} .
$$


(2) $\Phi_{v w}$ is the disjoint union of $\Phi_{w}$ and $\Phi_{v} w$ whenever $l(v w)=l(v)+l(w)$.

(3) $\Phi_{w^{-1}}=-\Phi_{w} w^{-1}$.

(4) $\Phi^{+}$is the disjoint union of $\Phi_{w}$ and $\Phi_{w_{0} w}$, where $w_{0}$ is the longest element of $W$.

Proof. Parts (1) and (2) are proved in 4. Part (3) follows from the definition as follows

$$
-\Phi_{w} w^{-1}=-\left(\Phi^{-} w \cap \Phi^{+}\right) w^{-1}=\Phi^{+} \cap \Phi^{-} w^{-1}=\Phi_{w^{-1}} .
$$

Finally, $\Phi$ is the disjoint union of $\Phi^{-} w$ and $\Phi^{+} w$, so $\Phi^{+}$is the disjoint union of $\Phi^{+} \cap \Phi^{-} w=\Phi_{w}$ and $\Phi^{+} \cap \Phi^{+} w=\Phi^{+} \cap \Phi^{-} w_{0} w=\Phi_{w_{0} w}$, and so we have (4).

2.3. Computation. We now give a construction of root data suitable for computation. Take $X$ and $Y$ to be $\mathbb{Z}^{d}$ with $e_{1}, \ldots, e_{d}$ and $f_{1}, \ldots, f_{d}$ both the standard basis. A root datum is determined by a pair of $n \times d$ integer matrices $A$ and $B$ such that the rows of $A$ are the simple roots and the rows of $B$ are the corresponding coroots; i.e., $A_{i j}=\left\langle\alpha_{i}, f_{j}\right\rangle$ and $B_{i j}=\left\langle e_{j}, \alpha_{i}^{\star}\right\rangle$. The Cartan matrix is $C=A B^{t}$.

Given a root $\beta$ and a simple root $\alpha_{i}$, knowledge of the Cartan matrix is sufficient to determine whether or not $\beta+\alpha_{i}$ is a root. Thus $\Phi^{+}$can be constructed recursively from the simple roots. The positive roots are constructed and stored in an order compatible with height, i.e., $h(\alpha)<h(\beta)$ implies that $\alpha<\beta$. We extend this to a linear ordering on $\Phi^{-} \cup\{0\} \cup \Phi^{+}$in the obvious way. The coroots are computed similarly.

\section{Structure COnstants}

We now briefly discuss the constants used to define semisimple Lie algebras and groups of Lie type and efficient methods for computing them. See [6] or [24] for more details.

Fix a root datum $\mathcal{R}=\left(X, \Phi, Y, \Phi^{\star}\right)$. For every pair of linearly independent roots $\alpha$ and $\beta$, we define $p_{\alpha \beta}$ to be the largest integer such that $-p_{\alpha \beta} \alpha+\beta$ is a root and we define $q_{\alpha \beta}$ to be the largest integer such that $q_{\alpha \beta} \alpha+\beta$ is a root. The vectors

$$
-p_{\alpha \beta} \alpha+\beta, \ldots,-\alpha+\beta, \beta, \alpha+\beta, \ldots, q_{\alpha \beta} \alpha+\beta
$$

are all roots; they form the chain through $\beta$ in the direction of $\alpha$. Furthermore $\left\langle\beta, \alpha^{\star}\right\rangle=p_{\alpha \beta}-q_{\alpha \beta}$.

The semisimple Lie algebra with root system $\Phi$ has basis elements $e_{\alpha}$, for $\alpha$ a root, and $h_{\alpha}$, for $\alpha$ a simple root, satisfying the relations

$$
\begin{aligned}
{\left[h_{\alpha}, h_{\beta}\right] } & =0, & {\left[e_{\alpha}, h_{\beta}\right] } & =\left\langle\alpha, \beta^{\star}\right\rangle e_{\alpha}, \\
{\left[e_{-\alpha}, e_{\alpha}\right] } & =\sum_{i=1}^{n}\left\langle\omega_{i}, \alpha^{\star}\right\rangle h_{\alpha_{i}}, & {\left[e_{\alpha}, e_{\beta}\right] } & = \begin{cases}N_{\alpha \beta} e_{\alpha+\beta} & \text { if } \alpha+\beta \in \Phi, \\
0 & \text { otherwise } .\end{cases}
\end{aligned}
$$

Chevalley [8] showed that the constants $N_{\alpha \beta}$ may be chosen to have the form $\varepsilon_{\alpha \beta}\left(p_{\alpha \beta}+1\right)$ where $\varepsilon_{\alpha \beta}= \pm 1$. This ensures that all the constants that appear in these relations are integral, so we can define a Lie algebra over $\mathbb{Z}$, and hence over an arbitrary field. For convenience, we take $N_{\alpha \beta}=0$ when $\alpha+\beta$ is not a root.

The constants $p_{\alpha \beta}, q_{\alpha \beta}$, and $\|\alpha\|^{2}$ can be computed directly from the root datum. Hence it only remains to compute the constants $\varepsilon_{\alpha \beta}$. From Theorem 4.1.2 of [6], 
we get the equations

$$
\begin{aligned}
& \varepsilon_{\alpha \beta}=-\varepsilon_{\beta \alpha}, \\
& \varepsilon_{\alpha \beta}=-\varepsilon_{-\alpha,-\beta}
\end{aligned}
$$

for arbitrary roots $\alpha$ and $\beta$;

$$
\begin{aligned}
\frac{N_{\alpha \beta}}{\|\gamma\|^{2}} & =\frac{N_{\beta \gamma}}{\|\alpha\|^{2}}=\frac{N_{\gamma \alpha}}{\|\beta\|^{2}}, \\
\varepsilon_{\alpha \beta} & =\varepsilon_{\beta \gamma}=\varepsilon_{\gamma \alpha}
\end{aligned}
$$

whenever $\alpha+\beta+\gamma=0$; and

$$
\frac{N_{\alpha \beta} N_{\gamma \delta}}{\|\alpha+\beta\|^{2}}+\frac{N_{\beta \gamma} N_{\alpha \delta}}{\|\beta+\gamma\|^{2}}+\frac{N_{\gamma \alpha} N_{\beta \delta}}{\|\gamma+\alpha\|^{2}}=0
$$

whenever $\alpha+\beta+\gamma+\delta=0$ and no pair is antipodal. Given a nonsimple positive root $\xi$, take the positive roots $\alpha, \beta$ such that $\xi=\alpha+\beta$ and $\alpha$ is as small as possible with respect to the ordering of Section 2.3 . We call $(\alpha, \beta)$ the extraspecial pair of $\xi$. We can choose the signs $\varepsilon_{\alpha \beta}$ on the extraspecial pairs arbitrarily and then all the structure constants are determined [6, Proposition 4.2.2]. This method gives us every possible system of structure constants; hence, if a user does hand calculations using a particular formula for the constants, it is possible to set the constants in our code to agree with those calculations. An algorithm for determining the structure constants from their values on extraspecial pairs is given in [16. Note that the number of extraspecial pairs is $N-n$ where $N$ is the number of positive roots. Furthermore $N$ is easily shown to be $O\left(n^{2}\right)$ by checking the number of roots for each of the classical types $A_{n}, B_{n}, C_{n}, D_{n}$.

In order to multiply elements in the Steinberg presentation efficiently, we must compute the structure constants very rapidly. Just storing the constants for the $O\left(n^{2}\right)$ extraspecial pairs and using the algorithm mentioned above is too slow. On the other hand, storing the constants for all $O\left(n^{4}\right)$ pairs uses too much memory. Hence we store the structure constants for pairs $(\alpha, \beta)$ with $0<\alpha<\beta$ and $\alpha+\beta \in$ $\Phi$, which we call special pairs. There are $O\left(n^{3}\right)$ such pairs, as can be shown by a case-by-case analysis for the classical types. We compute $\varepsilon_{\alpha \beta}$ for the special pairs in the following order: first by the height of $\xi=\alpha+\beta$, then by the position of $\alpha$ in the ordering of Section 2.3 , then by the position of $\beta$ in the same ordering. Take $\left(\alpha^{\prime}, \beta^{\prime}\right)$ to be the extraspecial pair of $\xi$. By (5) we have $\varepsilon_{\alpha \beta}=\operatorname{sign}\left(t_{1}-t_{2}\right)$ where

$$
t_{1}=\frac{N_{-\alpha^{\prime} \beta} N_{\beta^{\prime} \alpha}}{\left\|\beta-\alpha^{\prime}\right\|^{2}} \quad \text { and } \quad t_{2}=\frac{N_{-\alpha^{\prime} \alpha} N_{\beta^{\prime} \beta}}{\left\|\alpha-\alpha^{\prime}\right\|^{2}} .
$$

However $\beta^{\prime}$ may be larger than $\alpha$ so $N_{\beta^{\prime} \alpha}$ and $N_{\beta^{\prime} \beta}$ may not be among the constants we have already computed, and we would like to eliminate the negative root $-\alpha^{\prime}$ from these equations. If $\beta-\alpha^{\prime}=\beta^{\prime}-\alpha \notin \Phi$, then $t_{1}=0$; otherwise, by (3),

$$
\begin{aligned}
t_{1} & =\frac{\left\|\beta-\alpha^{\prime}\right\|^{2} N_{\alpha^{\prime}, \beta-\alpha^{\prime}}}{\|\beta\|^{2}} \cdot \frac{\left\|\beta-\alpha^{\prime}\right\|^{2} N_{\alpha^{\prime}, \beta^{\prime}-\alpha}}{\left\|\beta^{\prime}\right\|^{2}} \cdot \frac{1}{\left\|\beta-\alpha^{\prime}\right\|^{2}} \\
& =\frac{\left\|\beta-\alpha^{\prime}\right\|^{2}}{\|\beta\|^{2}\left\|\beta^{\prime}\right\|^{2}} N_{\alpha^{\prime}, \beta-\alpha^{\prime}} N_{\alpha, \beta^{\prime}-\alpha} ;
\end{aligned}
$$




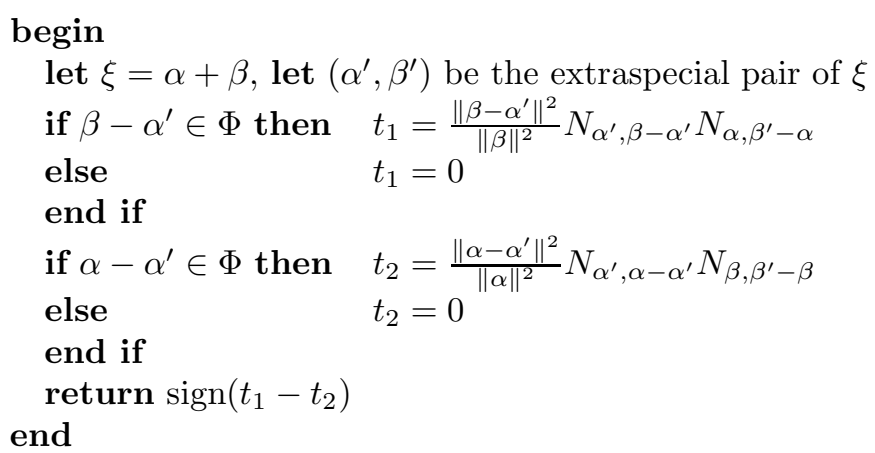

Algorithm 1. Computing $\varepsilon_{\alpha \beta}$ for special pairs given extraspecial pairs

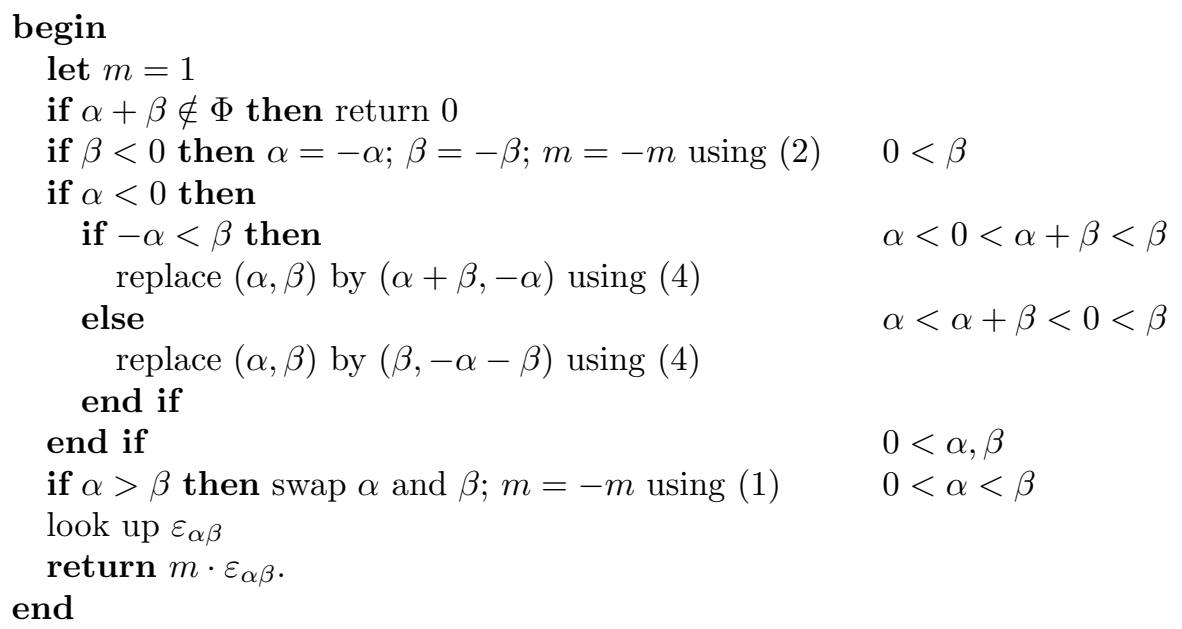

Algorithm 2. Computing $\varepsilon_{\alpha \beta}$ for all pairs given special pairs

both of the constants that appear here have already been computed. Similarly, if $\alpha-\alpha^{\prime}=\beta^{\prime}-\beta \notin \Phi$, then $t_{2}=0$; otherwise

$$
t_{2}=\frac{\left\|\alpha-\alpha^{\prime}\right\|^{2}}{\|\alpha\|^{2}\left\|\beta^{\prime}\right\|^{2}} N_{\alpha^{\prime}, \alpha-\alpha^{\prime}} N_{\beta, \beta^{\prime}-\beta}
$$

We can now cancel the common factor of $1 /\left\|\beta^{\prime}\right\|^{2}$ from $t_{1}$ and $t_{2}$ to get Algorithm 1

An alternative method is the cochain formula, which was developed for simply laced types by Frenkel and Kac [13] and Springer [27] and has been extended to all types by Rylands [25]. A modification of the cochain formula is given in [10] and has been implemented by him in both GAP [14] and Magma [2].

Once we have stored the constants for all special pairs, we need algorithms to compute them for an arbitrary pair of roots. Since these algorithms are called many times for every multiplication in our group, it is vital that they be as efficient as possible. In particular, we eliminate all recursive calls to improve the speed of the algorithm. In Algorithm 2 we compute $\varepsilon_{\alpha \beta}$ - similar methods are used to compute 
$N_{\alpha \beta}, p_{\alpha \beta}, q_{\alpha \beta}$ and the root chains, once they have been stored for all special pairs.

The following constants are now easily computed, for roots $\alpha, \beta$ and positive integers $i, j$ : whenever $(i-1) \alpha+\beta$ is a root, define

$$
M_{\alpha \beta i}=\left(\begin{array}{c}
p_{\alpha \beta}+i \\
i
\end{array}\right) \varepsilon_{\beta \alpha} \cdots \varepsilon_{(i-1) \alpha+\beta, \alpha} ;
$$

whenever $i \alpha+j \beta$ is a root, define

$$
C_{i j \alpha \beta}= \begin{cases}-M_{\alpha \beta i} & \text { for } j=1, \\ M_{\beta \alpha j} & \text { for } i=1, \\ -\frac{2}{3} M_{\alpha+\beta, \alpha, 2} & \text { for } i=3, j=2, \\ -\frac{1}{3} M_{\alpha+\beta, \beta, 2} & \text { for } i=2, j=3 ;\end{cases}
$$

and for $p=p_{\alpha \beta}$ and $q=q_{\alpha \beta}$ define

$$
\eta_{\alpha \beta}=(-1)^{p} \frac{\varepsilon_{\beta-p \alpha, \alpha} \cdots \varepsilon_{\beta-\alpha, \alpha}}{\varepsilon_{\beta-p \alpha, \alpha} \cdots \varepsilon_{\beta+(q-p-1) \alpha, \alpha}} .
$$

Note that these constants are also integral, thus allowing the presentations given in the next section to be defined for an arbitrary field.

\section{The Steinberg PResentation}

In this section we describe a presentation for the group of Lie type with root datum $\mathcal{R}=\left(X, \Phi, Y, \Phi^{\star}\right)$ over an arbitrary field $\mathbb{F}$. More details on this presentation can be found in, for example, [27].

4.1. The presentation. The Steinberg presentation for the group of Lie type $G_{\mathcal{R}}(\mathbb{F})$ has generators $x_{\alpha}(a)$, for $\alpha$ a root and $a \in \mathbb{F}$, and $y \otimes t$, for $y \in Y$ and $t \in \mathbb{F}^{\times}$. We also define auxiliary generators $n_{\alpha}=x_{\alpha}(1) x_{-\alpha}(-1) x_{\alpha}(1)$ corresponding to the reflections $s_{\alpha}$ in the Weyl group. The relations are

$$
\begin{aligned}
(y \otimes t)(y \otimes u) & =y \otimes(t u) \\
(y \otimes t)(z \otimes t) & =(y+z) \otimes t \\
\alpha^{\star} \otimes t & =x_{\alpha}(-1) x_{-\alpha}(1) x_{\alpha}(-1) \cdot x_{\alpha}(t) x_{-\alpha}\left(-t^{-1}\right) x_{\alpha}(t), \\
(y \otimes t)^{n_{\alpha}} & =y s_{\alpha}^{\star} \otimes t \\
x_{\alpha}(a) x_{\alpha}(b) & =x_{\alpha}(a+b) \\
x_{\alpha}(a)^{x_{\beta}(b)} & =x_{\alpha}(a) \prod_{i, j>0} x_{i \alpha+j \beta}\left(C_{i j \alpha \beta} a^{i} b^{j}\right) \\
x_{\alpha}(a)^{x_{-\alpha}(t)} & =x_{-\alpha}\left(-t^{2} a\right)^{x_{\alpha}\left(t^{-1}\right)}
\end{aligned}
$$

where $\alpha$ and $\beta$ are linearly independent roots, $y, z \in Y, a, b \in \mathbb{F}$ and $t, u \in \mathbb{F}^{\times}$. The product on the right-hand side of (11) runs over roots of the form $i \alpha+j \beta$ (for $i$ and $j$ positive integers) in the order described in Section 2.3. Relation (12) is redundant except when the rank is one.

4.2. Structure. The group $G_{\mathcal{R}}(\mathbb{F})$ is linear algebraic, even though we have described it abstractly. We have not used the presentations described in [7] or [30] because they define groups which are not necessarily algebraic when $\mathbb{F}$ is not algebraically closed.

The following result follows from Section 11.1 of [6]. 
Theorem 4.1. If $\mathcal{R}$ is irreducible, then $G_{\mathcal{R}}(\mathbb{F})^{\prime} / Z\left(G_{\mathcal{R}}(\mathbb{F})^{\prime}\right)$ is a simple group, unless $\mathcal{R}$ has type $A_{1}$ with $|\mathbb{F}| \leq 3$ or type $B_{2}$ or $G_{2}$ with $|\mathbb{F}|=2$.

More generally, suppose $G=G_{\mathcal{R}}(\mathbb{F})$ where $\mathcal{R}$ does not contain a summand of type $A_{1}$ with $|\mathbb{F}| \leq 3$ or of type $B_{2}$ or $G_{2}$ with $|\mathbb{F}|=2$. Then $G^{\prime} / Z\left(G^{\prime}\right)$ is a direct sum of simple groups. At the top we have $G / G^{\prime}=\left(Y \otimes \mathbb{F}^{\times}\right) /\left(\mathbb{Z} \Phi^{\star} \otimes \mathbb{F}^{\times}\right)$. At the bottom we have

$$
Z(G)=\left\{\prod_{i=1}^{n} f_{i} \otimes h_{i} \mid \prod_{i=1}^{n} h_{i}^{\left\langle x, f_{i}\right\rangle}=1 \text { for all } x \text { in } \mathbb{Z} \Phi\right\} .
$$

We define the following important subgroups of $G_{\mathcal{R}}(\mathbb{F})$.

- The maximal torus $H$ is the subgroup generated by the elements $y \otimes t$. It is isomorphic to the tensor product of the abelian groups $Y$ and $\mathbb{F}^{\times}$. Note that $Y$ is an additive group and $\mathbb{F}^{\times}$is a multiplicative group, so that, for instance, $n y \otimes t=y \otimes t^{n}$ for an integer $n$.

- $N$ is the subgroup generated by the maximal torus and the Weyl terms $n_{\alpha}$; it is the normaliser of $H$, except in certain cases where the field is small. For $w$ in the Weyl group $W$, take a reduced expression $w=s_{\beta_{1}} \cdots s_{\beta_{l}}$ and set $\dot{w}=n_{\beta_{1}} \cdots n_{\beta_{l}}$; this is well defined by Proposition 9.3.2 of [27]. There is an isomorphism between $N / H$ and $W$ given by $H \dot{w} \leftrightarrow w$.

- The unipotent subgroup $U$ is generated by the elements $x_{\alpha}(a)$ for $\alpha$ a positive root and $a \in \mathbb{F}$. Note that $U$ is a nilpotent group and, if $\mathbb{F}$ has characteristic $p, U$ is a $p$-group. For $w$ in $W$, define $U_{w}$ to be the subgroup of $U$ generated by the elements $x_{\alpha}(a)$ for $\alpha$ in $\Phi_{w}$ and $a \in \mathbb{F}$.

- The Borel subgroup $B=H U$. Its double cosets correspond to the elements of the Weyl group by $B \dot{w} B \leftrightarrow w$.

4.3. Example: Type $A_{n}$. Suppose $\mathcal{R}$ is a semisimple root datum of type $A_{n}$. Then the quotient $X / \mathbb{Z} \Phi$ is a subgroup of the cyclic group of order $n+1$; suppose it is the subgroup of size $d$ for some $d$ dividing $n+1$. The group of Lie type $G_{\mathcal{R}}(\mathbb{F})$ is isomorphic to $\mathrm{SL}_{n+1, d}(\mathbb{F}) / Z_{d}$, where

$$
\mathrm{SL}_{n+1, d}(\mathbb{F})=\left\{X \in \mathrm{GL}_{n+1}(\mathbb{F}) \mid \operatorname{det}(X)^{d}=1\right\}
$$

and

$$
Z_{d}=\left\{t I_{n+1} \mid t^{d}=1\right\}
$$

In particular, for $d=1$ we get $\mathrm{SL}_{n+1}(\mathbb{F})$ and for $d=n+1$ we get $\operatorname{PGL}_{n+1}(\mathbb{F})$. Note that some properties of these groups are dependent on the field. For example, if $\mathbb{F}$ is algebraically closed, then $\mathrm{PGL}_{n+1}(\mathbb{F})=\mathrm{PSL}_{n+1}(\mathbb{F})$ which is simple as an abstract group. In general $\mathrm{PGL}_{n+1}(\mathbb{F}) / \mathrm{PSL}_{n+1}(\mathbb{F}) \cong \mathbb{F}^{\times} /\left(\mathbb{F}^{\times}\right)^{n+1}$ and $\mathrm{PSL}_{n+1}(\mathbb{F})$ may not be algebraic.

\section{Arithmetic And Data Structures}

We now describe algorithms for element arithmetic in the group of Lie type $G=G_{\mathcal{R}}(\mathbb{F})$. A normal form for elements is a very useful tool for doing computations in a group. In the case of permutation groups, for example, we get a normal form from a base and strong generating set. In our case, we use the Bruhat decomposition [6, Corollary 8.4.4]: each $g \in G$ can be expressed uniquely in the form

$$
g=u h \dot{w} u^{\prime},
$$

where $u \in U, h \in H, w \in W$, and $u^{\prime} \in U_{w}$. 
5.1. Actions. The generators of $G$ are of three types: unipotent terms $x_{\alpha}(a)$, Weyl terms $n_{\alpha}$, and torus terms $y \otimes t$. The following equations describe how these generators act on each other, thus allowing us to exchange unipotent, Weyl and torus terms in a word. We also give equations for unipotent terms with negative roots. It is convenient to introduce the notation $h_{\alpha}(t)=\alpha^{\star} \otimes t$.

- The action of the Weyl terms on the maximal torus:

$$
\begin{aligned}
(y \otimes t)^{n_{\alpha}} & =y s_{\alpha}^{\star} \otimes t, \\
n_{\alpha}(y \otimes t) & =y s_{\alpha}^{\star} \otimes t .
\end{aligned}
$$

- The action of the Weyl terms on the unipotent subgroup:

$$
\begin{aligned}
& x_{\beta}(a)^{n_{\alpha}}=x_{\beta s_{\alpha}}\left(\eta_{\alpha, \beta} a\right), \\
& { }^{n_{\alpha}} x_{\beta}(a)=x_{\beta s_{\alpha}}\left(\eta_{\alpha, \beta}(-1)^{\left\langle\alpha, \beta^{\star}\right\rangle} a\right) .
\end{aligned}
$$

- The action of the maximal torus on the unipotent subgroup:

$$
\begin{aligned}
& x_{\beta}(a)^{y \otimes t}=x_{\beta}\left(t^{\langle\beta, y\rangle} a\right), \\
& y \otimes t \\
& x_{\beta}(a)=x_{\beta}\left(t^{-\langle\beta, y\rangle} a\right) .
\end{aligned}
$$

- Negative roots:

$$
\begin{aligned}
x_{-\alpha}(t) & =x_{\alpha}\left(t^{-1}\right) n_{\alpha} h_{\alpha}\left(-t^{-1}\right) x_{\alpha}\left(t^{-1}\right), \\
x_{\alpha}(t) n_{\alpha} & =x_{-\alpha}\left(t^{-1}\right) h_{\alpha}\left(-t^{-1}\right) x_{\alpha}\left(-t^{-1}\right) .
\end{aligned}
$$

We now discuss computation with torus elements, Weyl group representatives and unipotent elements in turn, before putting it all together in Section 5.5.

5.2. Torus elements. The torus is $H=Y \otimes \mathbb{F}^{\times}$. Recall that $Y$ has a basis $f_{1}, \ldots, f_{d}$, so every element of $Y \otimes \mathbb{F}^{\times}$can be written uniquely in the form $\prod_{i=1}^{d} f_{i} \otimes$ $h_{i}$ where $h_{i} \in \mathbb{F}^{\times}$. This element can be represented on the computer by the vector $\left(h_{1}, \ldots, h_{d}\right)$. Multiplication and inversion are just coordinatewise operations.

Given an automorphism of $Y$ whose matrix with respect to $f_{1}, \ldots, f_{d}$ is $M$, we get an induced automorphism of $Y \otimes \mathbb{F}^{\times}$:

$$
\prod_{i=1}^{d} f_{i} \otimes h_{i} \mapsto \prod_{i=1}^{d}\left(\sum_{j=1}^{d} M_{i j} f_{j}\right) \otimes h_{i}=\prod_{j=1}^{d} f_{j} \otimes\left(\prod_{i=1}^{d} h_{i}^{M_{i j}}\right)
$$

Using equation (13), we can compute the action of $n_{\alpha}$ on a torus element by taking $M$ to be the matrix of $s_{\alpha}^{\star}$.

A torus element is in the centre of $G$ if it is of the form $\prod_{i=1}^{n} f_{i} \otimes h_{i}$ with $\prod_{i=1}^{n} h_{i}^{\left\langle x, f_{i}\right\rangle}=1$ for all $x \in \mathbb{Z} \Phi$. This is equivalent to

$$
1=\prod_{i=1}^{d} h_{i}^{\left\langle\alpha_{j}, f_{i}\right\rangle}=\prod_{i=1}^{d} h_{i}^{A_{j i}}
$$

for $j=1, \ldots, n$. 
5.3. Weyl group representatives. Recall that the Weyl group $W$ is a Coxeter group and $\dot{w}=n_{\beta_{1}} \cdots n_{\beta_{l}}$ where $w$ has reduced expression $s_{\beta_{1}} \cdots s_{\beta_{l}}$. The following equations are useful for computation:

$$
\begin{aligned}
n_{\beta}{ }^{n_{\alpha}} & =n_{\beta s_{\alpha}} h_{\beta s_{\alpha}}\left(\eta_{\alpha, \beta}\right), \\
n_{\alpha}{ }^{-1} & =n_{-\alpha}=h_{\alpha}(-1) n_{\alpha}=n_{\alpha} h_{\alpha}(-1), \\
n_{\alpha}{ }^{2} & =h_{\alpha}(-1) .
\end{aligned}
$$

We compute products by repeated use of the formula

$$
\dot{w} n_{\alpha}= \begin{cases}\left(w s_{\alpha}\right)^{.} & \text {if } \alpha \notin \Phi_{w}, \alpha \text { simple } \\ h_{\alpha w^{-1}}(-1)\left(w s_{\alpha}\right) & \text { if } \alpha \in \Phi_{w}, \alpha \text { simple. }\end{cases}
$$

The inverse of $\dot{w}$ is computed as follows, using Lemma 2.1(1):

$$
\begin{aligned}
\dot{w}^{-1} & =\dot{s}_{\beta_{l}}^{-1} \cdots \dot{s}_{\beta_{2}}^{-1} \dot{s}_{\beta_{1}}^{-1} \\
& =n_{\beta_{l}} h_{\beta_{l}}(-1) \cdots n_{\beta_{2}} h_{\beta_{2}}(-1) \cdot n_{\beta_{1}} h_{\beta_{1}}(-1) \\
& =\left(n_{\beta_{l}} \cdots n_{\beta_{1}}\right) h_{\beta_{l} s_{\beta_{l-1}} \cdots s_{\beta_{1}}}(-1) \cdots h_{\beta_{2} s_{\beta_{1}}}(-1) h_{\beta_{1}}(-1) \\
& =\left(w^{-1}\right) \cdot \prod_{\alpha \in \Phi_{w^{-1}}} h_{\alpha}(-1) .
\end{aligned}
$$

Note that when $\alpha$ is a nonsimple root, $n_{\alpha}=h \dot{s}_{\alpha}$ where the torus element $h$ can be computed using (21) and the fact that $s_{\alpha}$ is conjugate to a simple reflection of $W$.

5.4. The unipotent subgroup. The unipotent subgroup $U$ is generated by the elements $x_{\alpha}(a)$ for $\alpha$ a positive root and $a \in \mathbb{F}$. It is shown in [6] that every element of $U$ can be written uniquely in the form $\prod_{\alpha \in \Phi^{+}} x_{\alpha}\left(a_{\alpha}\right)$ where the product is in the order described in Section 2.3. This proof is constructive and proceeds by collection using the commutator formulas (11) and (10). Computationally we use collection from the left to put a unipotent element into this normal form. This is generally the most efficient known collection algorithm 22] and our testing indicates that it is most efficient in this case as well. We invert a unipotent element by reversing the order of the terms and replacing the field elements by their negatives, then doing collection to put the terms back in the right order.

Given a Weyl group element $w$, Carter [6] proves that we can collect any unipotent element into the form

$$
\prod_{\alpha \in \Phi^{+} \backslash \Phi_{w}} x_{\alpha}\left(a_{\alpha}\right) \cdot \prod_{\alpha \in \Phi_{w}} x_{\alpha}\left(a_{\alpha}\right) .
$$

Note that $\prod_{\alpha \in \Phi_{w}} x_{\alpha}\left(a_{\alpha}\right)$ is a general element of the subgroup $U_{w}$. This is used to normalise an element of the form $u h \dot{w} u^{\prime}$ with $u^{\prime}$ in $U$ but not necessarily in $U_{w}$ : put $u^{\prime}$ in the form (25); then exchange the first part with $h \dot{w}$ using (15) and (17) and multiply $u$ by the result.

Finally note that we can easily collect all terms corresponding to a fixed $\operatorname{root} \alpha$, so every unipotent element can be put in the form

$$
x_{\alpha}(a) \cdot u^{\prime} \text { where no term of the form } x_{\alpha}\left(a^{\prime}\right) \text { appears in } u^{\prime} .
$$




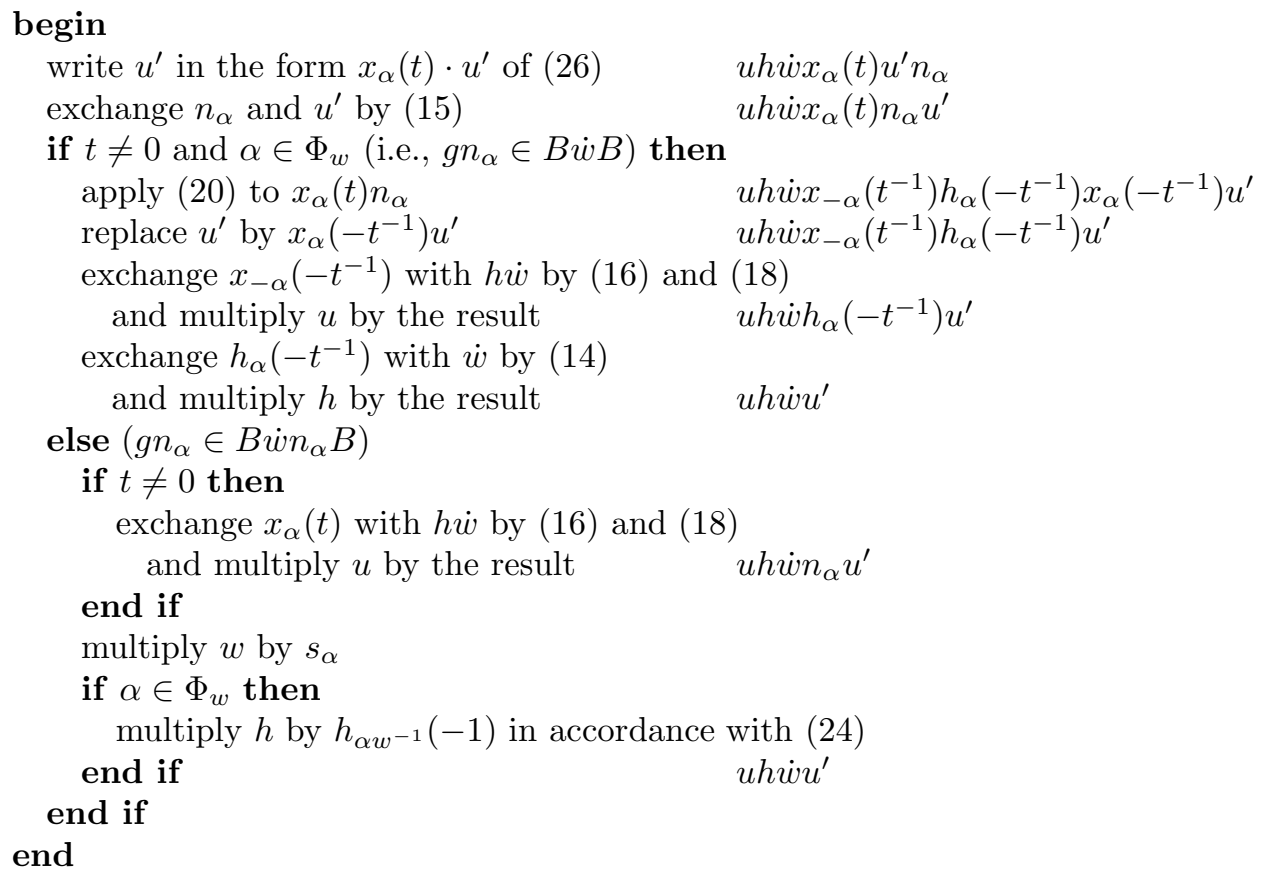

\section{Algorithm 3. Multiplication by a Weyl term}

5.5. Normalisation and arithmetic. Recall that we can exchange unipotent, Weyl and torus terms in a word using the equations in Section 5.1. Our goal is to efficiently use these equations to put a word into Bruhat normal form. It suffices to give an algorithm for normalising the product of a normalised element by a unipotent element, torus element or Weyl term. Note that we assume that all the terms $x_{\alpha}(t)$ which appear in our word have $\alpha$ positive - any negative roots that appear in the original word can be eliminated with equation (19). Much of the difficulty in this algorithm is ensuring that the roots remain positive when we exchange unipotent terms with Weyl terms.

Suppose $g=u h \dot{w} u^{\prime}$ is in normal form. To multiply $g$ by a torus element, exchange the torus element with $\dot{w} u^{\prime}$ using (17) and (14), then multiply $h$ by the result. To multiply $g$ by a unipotent element, multiply $u^{\prime}$ by the unipotent element and normalise $u h \dot{w} u^{\prime}$ as in the previous subsection.

Finally consider the more complicated case of multiplication by a Weyl term $n_{\alpha}$. Recall that

$$
g n_{\alpha}=u h \dot{w} u^{\prime} n_{\alpha} \in B \dot{w} B n_{\alpha} \subseteq B \dot{w} n_{\alpha} B \cup B \dot{w} B,
$$

where the union is disjoint. This gives us the two main cases of Algorithm 3 In order to clarify the pseudocode, we write the word we are working on to the right, but note that the values of the symbols $u, h, w$ and $u^{\prime}$ change from line to line.

It is now easy to normalise the product of two normalised words. We invert a normalised word by inverting each term and reversing their order, then swapping $h$ and $\dot{w}$ and normalising the unipotent parts as in Section 5.4. We can do conjugation more efficiently using the formulas of Section 5.1 directly rather than computing the product $y^{-1} x y$, but the result still needs to be normalised. Commutators are 
computed with one inversion, one conjugation, and one multiplication: $[x, y]=$ $x^{-1} \cdot x^{y}$.

Note that the most time-consuming part of these arithmetic operations is collecting unipotent elements. Collection is also the only part of this algorithm which is not known to run in polynomial time. In Section 8.1 we show that there is a provably polynomial time alternative to collection, which nevertheless seems to be slower in practice.

\section{Computing linear Representations}

Computing an irreducible highest weight representation for a group of Lie type is fairly straightforward since the representations for semisimple Lie algebras can already be computed by the algorithm of de Graaf [11]. Let $G$ be a group of Lie type, so the derived group $G^{\prime}$ is a semisimple group. Let $L$ be the integral Lie algebra corresponding to $G^{\prime}$ and let $\rho: L \rightarrow \mathfrak{g l}(V)$ be the irreducible highest weight representation on the free $\mathbb{Z}$-module $V$ with highest weight $\lambda \in X$. We define $x_{\alpha}(T)$ as $\exp \left(T \rho\left(e_{\alpha}\right)\right) \in \operatorname{End}_{\mathbb{Z}[T]}(V)$, where $T$ is an indeterminate. The matrix for $x_{\alpha}(a)$ for $a \in \mathbb{F}$ is simply gotten by substitution; this acts on the $\mathbb{F}$-vector space $V \otimes_{\mathbb{Z}} \mathbb{F}$. We also need matrices for the elements of the torus $Y \otimes \mathbb{F}^{\times}$which are not contained in $\mathbb{Z} \Phi^{\star} \otimes \mathbb{F}^{\times}$- this requires a linear algebra calculation the details of which we omit.

This algorithm runs in polynomial time in the coefficients of $\lambda$ for a fixed group $G$. It is not possible for the algorithm to be polynomial in the rank of $G$ and the coefficients of $\lambda$, since the dimension of the irreducible highest weight module is not polynomial in these parameters.

The efficiency of this computation can be improved by noticing that the matrices corresponding to terms in the Steinberg presentation are sparse. So rather than multiplying on the left or right by the matrices, we use the corresponding row or column operations. This observation motivates the algorithm of the next section.

\section{Generalised ROW AND COLUmn REDUCTION}

We now consider the converse problem of converting from a representation to the Steinberg presentation. Together with the previous section, this allows us to transfer between representations and the Steinberg presentation, so we can use whichever is most appropriate for the computation at hand.

Our algorithm is a generalisation of row and column reduction or, more specifically, the LUP algorithm. It works for any nontrivial quotient of a highest weight representation. In many cases it can be extended to an arbitrary representation: First note that we can reduce to the case of an irreducible representation by working our way down a composition series of the module. If $\mathbb{F}$ is algebraically closed or finite, then every irreducible module is a quotient of a highest weight module.

We assume that $G$ is a group of Lie type $G_{\mathcal{R}}(\mathbb{F})$ and that $\rho: G \rightarrow \operatorname{GL}(V)$ is a nontrivial quotient of a highest weight representation on the $\mathbb{F}$-space $V$. We consider $V$ to be a $G$-module by $v g:=v \rho(g)$. Let $A$ be a matrix in $\mathrm{GL}(V)$; to simplify our exposition, we assume that $A \in \rho(G)$ (in fact, our algorithm is easily converted into a membership test for $\rho(G)$; see Theorem 8.1). Our aim is to find $g \in G$ so that $A=\rho(g)$, where $g$ is given as a word in Bruhat normal form.

7.1. The case of $\mathrm{SL}_{2}(\alpha)$. Given a root $\alpha$, define $\mathrm{SL}_{2}(\alpha)$ to be the subgroup of $G$ generated by the elements $x_{\alpha}(a)$ and $x_{-\alpha}(a)$ for all $a \in \mathbb{F}$. This subgroup 
is isomorphic to a nontrivial quotient of $\mathrm{SL}_{2}(\mathbb{F})$. We define a (projective linear) action of $\mathrm{SL}_{2}(\alpha)$ on the polynomial algebra $\mathbb{F}[x, y]$ by

$$
\begin{array}{rll}
x_{\alpha}(a): & x \mapsto x, & y \mapsto a x+y ; \\
x_{-\alpha}(a): & x \mapsto x+a y, & y \mapsto y ; \\
n_{\alpha}: & x \mapsto-y, & y \mapsto x ; \\
h_{\alpha}(t): & x \mapsto t x, & y \mapsto t^{-1} y .
\end{array}
$$

The highest weight module for $\mathrm{SL}_{2}(\alpha)$ of weight $m$ is just the submodule of $\mathbb{F}[x, y]$ consisting of homogeneous polynomials of degree $m$; we denote this module by $V(m)$. Note that, since $\mathrm{SL}_{2}(\alpha)$ is a quotient of $\mathrm{SL}_{2}(\mathbb{F})$, this may be a projective representation - for our purposes, however, this is not a problem. If $\mathbb{F}$ has characteristic $0, V(m)$ is irreducible. If $\mathbb{F}$ has characteristic $p$, then we get an irreducible module by quotienting out the submodule spanned by the terms $x^{i} y^{m-i}$ for which $p$ divides $\left(\begin{array}{c}m \\ i\end{array}\right)$.

Every irreducible $\mathrm{SL}_{2}(\alpha)$-module $M$ is isomorphic to a nontrivial quotient of $V(m)$ for some $m$. Suppose we are given such a module $M$, we now show how to compute the value of $m$. Let $v$ be a nonzero fixed vector of $x_{\alpha}(1)$. Then $v$ can be considered to be the image of $x^{m}$ and we can compute $m$ with the formula $v h_{\alpha}(t)=t^{m} v$. If $\mathbb{F}$ is finite of size $q$, then we can assume that $m<q$ by the Steinberg tensor product theorem 29] and so $m$ is the smallest positive integer satisfying this formula for $t$ a primitive element of $\mathbb{F}$. We also know that $(-1)^{m} v n_{\alpha}$ is the image of $y^{m}$ and the images of the subspaces $\mathbb{F} x^{i} y^{m-i}$ are found by taking eigenspaces of $h_{\alpha}(t)$. Hence we can compute an inner product . on $V$ so that these one-dimensional subspaces are orthogonal, and $v$ and $v n_{\alpha}$ have norm one.

Given a matrix $A \in \mathrm{GL}(V)$, we want to find $a, b \in \mathbb{F}, t \in \mathbb{F}^{\times}$and $w \in W=$ $\left\{1, s_{\alpha}\right\}$ such that $A=\rho\left(x_{\alpha}(a) h_{\alpha}(t) \dot{w} x_{\alpha}(b)\right)$. By an easy computation, we get

$$
\begin{array}{ll}
x^{m} g \cdot y^{m}=(-t)^{m}, & x^{m} g \cdot x^{m}=(-b t)^{m} ; \\
x^{m} h \cdot y^{m}=0, & x^{m} h \cdot x^{m}=t^{m}, \quad y^{m} h \cdot x^{m}=(a t)^{m}
\end{array}
$$

where $g=x_{\alpha}(a) h_{\alpha}(t) n_{\alpha} x_{\alpha}(b)$ and $h=x_{\alpha}(a) h_{\alpha}(t)$. Hence we have Algorithm 4 using the fact that $v$ is the image of $x^{m}$ and $(-1)^{m} v n_{\alpha}$ is the image of $y^{m}$.

\subsection{Computing weights and weight spaces. Given $\mu \in X$, we define}

$$
V_{\mu}=\left\{v \in V \mid v(y \otimes t)=t^{\langle\mu, y\rangle} v \text { for all } y \in Y, t \in \mathbb{F}^{\times}\right\} .
$$

Then $V=\bigoplus_{\mu} V_{\mu}$ and we call $\mu$ a weight of $V$ if $V_{\mu}$ is nontrivial. The dominance order for weights is defined by $\mu \preceq \nu$ if $\nu-\mu=\sum_{i=1}^{n} m_{i} \alpha_{i}$ where every $m_{i} \geq 0$; we write $\mu \prec \nu$ when $\mu \preceq \nu$ and $\mu \neq \nu$. Since $V$ is a nontrivial quotient of a highest weight representation, it has a dominance-highest weight $\lambda$ and $V_{\lambda}$ is one dimensional. In many applications, $\lambda$ and $V_{\lambda}$ are assumed to be known. However they can be computed if necessary: $V_{\lambda}$ is the intersection of the fixed spaces of the matrices $\rho\left(x_{\alpha}(1)\right)$ for $\alpha$ a simple root. The highest weight can be computed using the formula $\lambda=\sum_{i=1}^{n}\left\langle\lambda, \alpha_{i}^{\star}\right\rangle \omega_{i}$ and the fact that the $\mathrm{SL}_{2}\left(\alpha_{i}\right)$-submodule generated by $V_{\lambda}$ is a nontrivial quotient of $V(m)$ with $m=\left\langle\lambda, \alpha_{i}^{\star}\right\rangle$. 


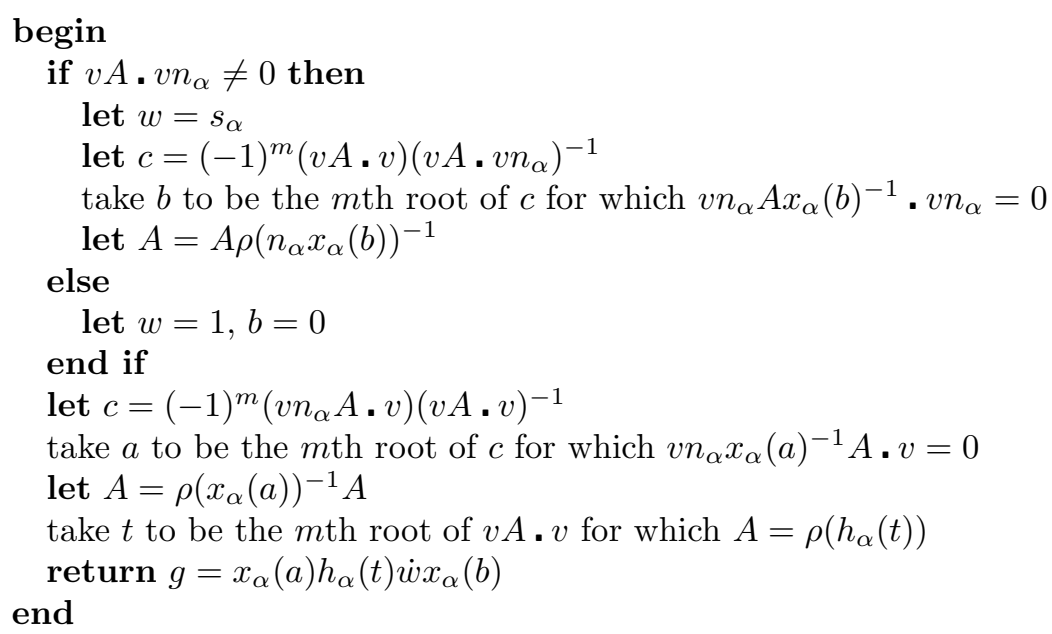

Algorithm 4. The $\mathrm{SL}_{2}(\alpha)$ algorithm

We frequently use the following basic facts about the action of $G$ on $V$ :

$$
\begin{aligned}
V_{\mu} x_{\alpha}(t) & \subseteq \bigoplus_{\nu \succeq \mu} V_{\nu} \quad \text { for } \alpha \in \Phi^{+}, \\
V_{\mu} \dot{w} & =V_{\mu w}, \\
V_{\mu} H & =V_{\mu} .
\end{aligned}
$$

Fix a nonzero vector $v_{\lambda}$ in $V_{\lambda}$. Write $\Omega$ for the orbit of $\lambda$ under the action of $W$; for each $\mu \in \Omega$, choose some $w \in W$ so that $\mu=\lambda w$ and fix $v_{\mu}=v_{\lambda} \dot{w}$. Note that $V_{\mu}=\mathbb{F} v_{\mu}$ and $v_{\mu}$ is well defined up to a sign. We can compute $V_{\mu}$ for a weight $\mu \notin \Omega$ by taking eigenspaces of torus elements. This allows us to compute a bilinear form . on $V$ for which the vectors $v_{\mu}$ for $\mu \in \Omega$ are orthonormal, and $\sum_{\mu \notin \Omega} V_{\mu}$ is contained in the radical.

By Theorem 29 of [30], there is an automorphism of $G$ such that

$$
x_{\alpha}(a) \mapsto x_{-\alpha}\left(\varepsilon_{\alpha} a\right), \quad(y \otimes t) \mapsto y \otimes t^{-1}
$$

provided the signs $\varepsilon_{\alpha}$ satisfying the equation

$$
\varepsilon_{\alpha} \varepsilon_{\beta}=-\varepsilon_{\alpha+\beta}
$$

whenever $\alpha+\beta$ is a root. We can now define an antiautomorphism $\delta$ on $G$ by composition of inversion in $G$ with the above automorphism, so that

$$
x_{\alpha}(a)^{\delta}=x_{-\alpha}\left(\varepsilon_{\alpha} a\right)^{-1}=x_{-\alpha}\left(-\varepsilon_{\alpha} t\right), \quad(y \otimes t)^{\delta}=y \otimes t .
$$

We want the additional property that $\delta^{2}=1$, which is true if and only if

$$
\varepsilon_{-\alpha}=\varepsilon_{\alpha}
$$

for every root $\alpha$. It is easily shown that $H \dot{w}^{\delta}=H \dot{w}^{-1}$. We take all $\varepsilon_{\alpha}=-1$ : clearly (30) is satisfied, so $\delta$ is an antiautomorphism, and (31) is satisfied, so it is an involution. 
We define a left action of $G$ on $V$ by $g v=v g^{\delta}$; we wish to show that this has the property that

$$
u g \cdot v=u \cdot g v .
$$

It suffices to take $u$ and $v$ of the form $v_{\mu}$ with $\mu \in \Omega$, since $\sum_{\sigma \notin \Omega} V_{\sigma}$ is in the radical. Furthermore, it suffices to take $g$ to be a generator, either a torus term or a unipotent term. For a torus term $g=y \otimes t$, both sides are zero unless $u=v=v_{\mu}$ and $v_{\mu}(y \otimes t) \cdot v_{\mu}=t^{\langle\mu, y\rangle}=v_{\mu} \cdot v_{\mu}(y \otimes t)^{\delta}$. For a unipotent term $g=x_{\alpha}(a)$, both sides are zero unless $u=v=v_{\mu}$, or $u=v_{\mu}$ and $v=v_{\nu}$ with $\nu=\mu s_{\alpha}$. As in Section 7.1 the $\mathrm{SL}_{2}(\alpha)$-module generated by $v_{\mu}$ can be identified with a quotient of $V(m)$ with $m=\left\langle\mu, \alpha^{\star}\right\rangle$. The vector $v_{\mu}$ is identified with $x^{m}$, and $v_{\nu} n_{\alpha}=e v_{\mu}$ $(e= \pm 1)$ is identified with $y^{m}$. Now

$$
\begin{aligned}
v_{\mu} x_{\alpha}(a) & =e\left(v_{\nu} n_{\alpha}\right) x_{\alpha}(a) \\
& =e y^{m} x_{\alpha}(a) \\
& =e(y+a x)^{m} \in v_{\mu}+e a^{m} v_{\nu}+\sum_{\sigma \notin \Omega} V_{\sigma}
\end{aligned}
$$

and

$$
\begin{aligned}
v_{\nu} x_{\alpha}(a)^{\delta} & =x^{m} x_{-\alpha}(a) \\
& =(x+a y)^{m} \in v_{\nu}+e a^{m} v_{\mu}+\sum_{\sigma \notin \Omega} V_{\sigma} .
\end{aligned}
$$

Consequently, if $u=v=v_{\mu}$, then

$$
u g \cdot v=v_{\mu} x_{\alpha}(a) \cdot v_{\mu}=1=v_{\mu} \cdot v_{\mu} x_{\alpha}(a)=u \cdot v g^{\delta}=u \cdot g v,
$$

and if $u=v_{\mu}$ and $v=v_{\nu}$, then

$$
u g \cdot v=v_{\mu} x_{\alpha}(a) \cdot v_{\nu}=e a^{m}=v_{\mu} \cdot v_{\nu} x_{\alpha}(a)=u \cdot v g^{\delta}=u \cdot g v,
$$

so we are done.

7.3. The inductive step. In this section we show how to reduce from $G$ to a reductive subgroup of smaller semisimple rank.

Since $\lambda$ is a dominant weight, its stabiliser $W_{\lambda}$ is actually the standard parabolic subgroup $W_{J}$ with $J=\left\{s \mid\left\langle\lambda, \alpha_{s}^{\star}\right\rangle=0\right\}$. Within $G$ we have opposite parabolic subgroups $P_{J}=B \dot{W}_{J} B$ and $P_{J}^{\delta}=B^{\delta} \dot{W}_{J} B^{\delta}$. The Levi complement $L_{J}=P_{J} \cap P_{J}^{\delta}$ is reductive with root datum $\left(X, \Phi_{\lambda}, Y, \Phi_{\lambda}^{\star}\right)$, where

$$
\Phi_{\lambda}=\left\{\alpha \in \Phi \mid\left\langle\lambda, \alpha^{\star}\right\rangle=0\right\}=\Phi_{J}
$$

and $\Phi_{\lambda}^{\star}=\left\{\alpha^{\star} \mid \alpha \in \Phi_{\lambda}\right\}$. In particular, the semisimple rank of $L_{J}$ is strictly smaller than the semisimple rank of $G$ and the Weyl group of $L_{J}$ is $W_{J}=W_{\lambda}$.

Proposition 7.1. Let $g$ be an element of $G$. Then $g \in L_{J}$ if, and only if, $V_{\lambda} g=V_{\lambda}$ and $g V_{\lambda}=V_{\lambda}$.

Proof. First we show that $g \in P_{J}$ if, and only if, $V_{\lambda} g=V_{\lambda}$. Since $\lambda$ is the highest weight, $V_{\lambda} B=V_{\lambda}$ and so $V_{\lambda} B \dot{w} B=V_{\lambda w} B=\sum_{\mu \succeq \lambda w} V_{\mu}$ by (27) and (29). Clearly this is equal to $V_{\lambda}$ exactly when $\lambda w=\lambda$, i.e., $w \in W_{J}$.

On the other hand, $g V_{\lambda}=V_{\lambda}$ means $V_{\lambda} g^{\delta}=V_{\lambda}$ which is equivalent to $g^{\delta} \in P_{J}$, i.e., $g \in P_{J}^{\delta}$. Since $L_{J}=P_{J} \cap P_{J}^{\delta}$, we are done. 
Any element $w$ of $W$ can be decomposed in the form $w_{J} w^{J}$ where $w_{J} \in W_{J}$ and $w^{J}$ is the unique $W_{J}$-reduced element of $W$ such that $\lambda w^{J}=\lambda w$. We write $W^{J}$ for the set of all $W_{J}$-reduced elements of $W$. By Lemma 2.1(2), $\Phi_{w}$ is a disjoint union of $\Phi_{w_{J}} w^{J}$ and $\Phi_{w^{J}}$, and so we get a unique decomposition $U_{w}=\left(U_{w_{J}}\right)^{\dot{w}^{J}} U_{w^{J}}$. We are particularly interested in the factorisation $w_{0}=w_{0 J} w_{0}^{J}$ of the longest word in $W$. Now $w_{0 J}$ is the longest word of $W_{J}$. Since $w_{0 J}=w_{0}\left(w_{0}^{J}\right)^{-1}$, Lemma 2.1 4 ) implies that $\Phi^{+}$is the disjoint union of $\Phi_{\left(w_{0}^{J}\right)^{-1}}$ and $\Phi_{w_{0 J}}$ so we get a unique decomposition $U=U_{\left(w_{0}^{J}\right)^{-1}} U_{w_{0 J}}$. We can now modify the Bruhat decomposition as follows:

$$
\begin{aligned}
G & =\bigcup_{w \in W} U H \dot{w} U_{w} \\
& =\bigcup_{w_{J} \in W_{J}, w^{J} \in W^{J}} U_{\left(w_{0}^{J}\right)^{-1}} U_{w_{0 J}} H \dot{w}_{J} \dot{w}^{J} U_{w_{J}}^{\dot{w}^{J}} U_{w^{J}} \\
& =\bigcup_{w^{J} \in W^{J}} U_{\left(w_{0}^{J}\right)^{-1}}\left(\bigcup_{w_{J} \in W_{J}} U_{w_{0 J}} H \dot{w}_{J} U_{w_{J}}\right) \dot{w}^{J} U_{w^{J}} \\
& =\bigcup_{w^{J} \in W^{J}} U_{\left(w_{0}^{J}\right)^{-1}} L_{J} \dot{w}^{J} U_{w^{J}} .
\end{aligned}
$$

Recall that we are given an element $A \in \rho(G)$ and we wish to find a preimage $g \in G$. The first step is to find the value of $w^{J}$ in this decomposition of $g$; then we can find $u \in U_{\left(w_{0}^{J}\right)^{-1}}$ and $u^{\prime} \in U_{w^{J}}$ so that $A \in \rho\left(u L_{J} \dot{w}^{J} u^{\prime}\right)$. The problem is then reduced to finding a word for $\rho(u)^{-1} A \rho\left(\dot{w}^{J} u^{\prime}\right)^{-1}$ in the reductive subgroup $L_{J}$.

Since $w^{J}$ is the unique $W_{J}$-reduced element of $W$ with $\lambda w^{J}=\lambda w$, it can be found using the following lemma.

Lemma 7.2. Let $g$ be an element of the double coset $B \dot{w} B$ of $G$. If $\mu$ is the minimal element of $\Omega$ such that $v_{\lambda} g \cdot v_{\mu} \neq 0$, then $\lambda w=\mu$.

Proof. This follows from the facts that

$$
V_{\lambda} B \dot{w} B \subseteq \sum_{\mu \succeq \lambda w} V_{\mu}
$$

and

$$
v_{\lambda} g \cdot v_{\lambda w} \neq 0
$$

We now know that $A=\rho(g)$ with $g \in U_{\left(w_{0}^{J}\right)^{-1}} L_{J} \dot{w}^{J} U_{w^{J}}$ for fixed $w^{J}$. Write $g=u h \dot{w}^{J} u^{\prime}$ where $u \in U_{\left(w_{0}^{J}\right)^{-1}}, h \in L_{J}$, and

$$
u^{\prime}=\prod_{\alpha \in \Phi_{w^{J}}} x_{\alpha}\left(a_{\alpha}\right) \in U_{w^{J}} .
$$

Let $\gamma$ be a dominance minimal root in $\Phi_{w^{J}}$ with $a_{\gamma} \neq 0$. Using (26), we can rewrite $u^{\prime}$ in the form

$$
\prod_{\alpha \in \Phi_{w^{J}}, \alpha \npreceq \gamma} x_{\alpha}\left(a_{\alpha}\right) x_{\gamma}\left(a_{\gamma}\right),
$$

with different values of $a_{\alpha}$ but with $a_{\gamma}$ still nonzero. As in Section [7.1 the $\mathrm{SL}_{2}(\gamma)$-module generated by $v_{\mu}$ can be identified with a quotient of $V(m)$ with 
$m=-\left\langle\mu, \gamma^{\star}\right\rangle$, the vector $v_{\mu}$ with the image of $y^{m}$, and $v_{\lambda} n_{\mu}$ with the image of $x^{m}$. Now

$$
\begin{aligned}
v_{\lambda} A=v_{\lambda} g & =v_{\lambda} u h \dot{w}^{J} u^{\prime}=v_{\lambda} h \dot{w}^{J} u^{\prime} \\
& \in \mathbb{F}^{\times} v_{\mu} u^{\prime} \\
& =\mathbb{F}^{\times} v_{\mu}\left(\prod_{\alpha \in \Phi_{w^{J}}, \alpha \npreceq \gamma} x_{\alpha}\left(a_{\alpha}\right)\right) x_{\gamma}\left(a_{\gamma}\right) \\
& \subseteq \mathbb{F}^{\times}\left(v_{\mu}+\sum_{\xi \in \mathbb{Z}_{\geq 0} \Phi_{w^{J} \backslash 0}} V_{\mu+\xi}\right) x_{\gamma}\left(a_{\gamma}\right) \\
& \subseteq \mathbb{F}^{\times}\left(a_{\gamma}^{m} v_{\mu} n_{\gamma}+m a_{\gamma}^{m-1} x^{m-1} y+\cdots+v_{\mu}\right)+\sum_{\nu \npreceq \mu s_{\gamma}} V_{\nu} .
\end{aligned}
$$

The final line follows from the fact that the only weights that can appear are of the form $\nu=\mu+\xi-i \gamma$ where $i=0, \ldots, m$ and $\xi$ is a nonzero linear combination of roots $\alpha \in \Phi_{w^{J}}, \alpha \npreceq \gamma$ with nonnegative coefficients. Such weights satisfy $\nu \npreceq$ $\mu-m \gamma=\mu s_{\gamma}$. We can now compute $a_{\gamma}$ by setting

$$
c=\left(v_{\lambda} A \cdot v_{\mu} n_{\gamma}\right)\left(v_{\lambda} A \cdot v_{\mu}\right)^{-1}
$$

and taking $a_{\gamma}$ to be the $m$ th root of $c$ with the property that $v_{\lambda} A x_{\gamma}\left(a_{\gamma}\right)^{-1} \cdot v_{\mu} n_{\gamma}=0$. By repeating this process for all the roots in $\Phi_{w^{J}}$ in an order compatible with the dominance order, we can compute the value of $u^{\prime}$. The order $<$ of Section 2.3 has the required property.

We now have $g=u h \dot{w}^{J}$ and we wish to find $u \in U_{\left(w_{0}^{J}\right)^{-1}}$. This is similar to the preceding case. We may write $u=x_{\gamma}\left(b_{\gamma}\right) \prod_{\beta \in \Phi_{\left(w_{0}^{J}\right)^{-1}}, \beta \succeq \gamma} x_{\beta}\left(b_{\beta}\right)$ and then

$$
\begin{aligned}
A v_{\mu}=g v_{\mu} & =u h \dot{w}^{J} v_{\mu} \\
& \in \mathbb{F}^{\times} u v_{\lambda}=\mathbb{F}^{\times} v_{\lambda} u^{\delta} \\
& =\mathbb{F}^{\times} v_{\lambda}\left(\prod_{\beta \in \Phi_{w}^{J}, \alpha \succeq \gamma} x_{-\beta}\left(b_{\beta}\right)\right) x_{-\gamma}\left(b_{\gamma}\right) \\
& \subseteq \mathbb{F}^{\times}\left(v_{\lambda}+\cdots+b_{\gamma}^{m} v_{\lambda} n_{\gamma}\right)+\sum_{\nu \succeq \mu s_{\gamma}} V_{\nu}
\end{aligned}
$$

where $m=\left\langle\lambda, \gamma^{\star}\right\rangle$. Hence we compute $b_{\gamma}$ by taking

$$
c=\left(v_{\lambda} n_{\gamma} \cdot A v_{\mu}\right)\left(v_{\lambda} \cdot A v_{\mu}\right)^{-1}
$$

and computing the $m$ th root $b_{\gamma}$ of $c$ such that $v_{\lambda} n_{\gamma} \cdot x_{\gamma}\left(b_{\gamma}\right)^{-1} A v_{\mu}=0$.

We now have all the ingredients required for our algorithm, which we describe in detail in the next two sections.

7.4. A base for the Weyl group. By iterating the procedure of the previous section, we get a sequence of reductive subgroups

$$
G=L_{J_{1}} \geq L_{J_{2}} \geq \cdots \geq L_{J_{k+1}}=H
$$

where

$$
\{1, \ldots, n\}=J_{1} \supseteq J_{2} \supseteq \cdots \supseteq J_{k+1}=\emptyset .
$$




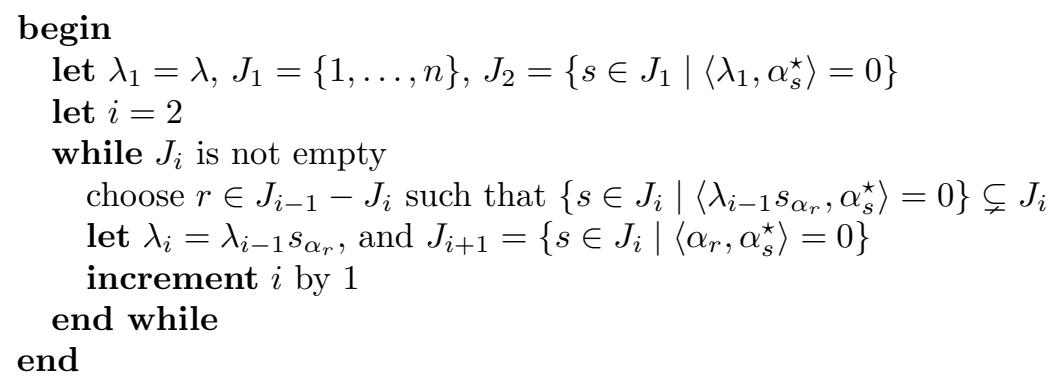

Algorithm 5. Computing a base of weights

Note that this chain of subgroups depends only on $G$, not on the particular matrix $A$. The subsets $J_{i}$ and the highest weight for each $L_{J_{i}}$ can be computed beforehand using the following theorem.

Theorem 7.3. Let $G$ be a reductive group with highest weight representation $V$. Consider the Weyl group as a permutation group on the weights in $\Omega$. Then $W$ has a base $\lambda_{1}, \ldots, \lambda_{k}$ with the following properties:

(1) $W_{\lambda_{1}, \ldots, \lambda_{i-1}}=W_{J_{i}}$ for some $J_{i} \subseteq\{1, \ldots, n\}$ and

(2) $\lambda_{i}$ is a highest weight for $L_{J_{i}}=B \dot{W}_{J_{i}} B$ acting on $V$.

Proof. We begin by putting $\lambda_{1}=\lambda, J_{1}=\{1,2, \ldots, n\}$ and $J_{2}=\left\{s \in J_{1}\right.$ | $\left.\left\langle\lambda_{1}, \alpha_{s}^{\star}\right\rangle=0\right\}$, and we proceed by induction on $i$. Given $\lambda_{1}, \lambda_{2}, \ldots, \lambda_{i-1}$ and $J_{1}, J_{2}, \ldots, J_{i}$ satisfying properties (1) and (2) with $J_{i} \neq \emptyset$, we find $r \notin J_{i}$ such that $\left\langle\lambda_{i-1} s_{\alpha_{r}}, \alpha_{s}^{\star}\right\rangle \neq 0$ for some $s \in J_{i}$. Put $\lambda_{i}=\lambda_{i-1} s_{\alpha_{r}}$. Then for all $s \in J_{i}$ we have

$$
\begin{aligned}
\left\langle\lambda_{i}, \alpha_{s}^{\star}\right\rangle & =\left\langle\lambda_{i-1} s_{\alpha_{r}}, \alpha_{s}^{\star}\right\rangle \\
& =\left\langle\lambda_{i-1}-\left\langle\lambda_{i-1}, \alpha_{r}^{\star}\right\rangle \alpha_{r}, \alpha_{s}^{\star}\right\rangle \\
& =-\left\langle\lambda_{i-1}, \alpha_{r}^{\star}\right\rangle\left\langle\alpha_{r}, \alpha_{s}^{\star}\right\rangle \geq 0 .
\end{aligned}
$$

Thus $J_{i+1}=\left\{s \in J_{i} \mid\left\langle\alpha_{r}, \alpha_{s}^{\star}\right\rangle=0\right\}=W_{\lambda_{1}, \ldots, \lambda_{i}}$ and $\lambda_{i}$ satisfy properties (1) and (2).

The weights $\lambda_{i}$ and sets $J_{i}$ are constructed using Algorithm [5. Suppose that $w$ is an element of the Weyl group $W$. Then we have a unique factorisation $w=$ $w_{k} \cdots w_{2} w_{1}$ where $w_{i}$ is the unique $W_{J_{i+1}}$-reduced element of $W_{J_{i}}$ such that $\lambda_{i} w_{i}=$ $\lambda_{i} w w_{1}^{-1} \cdots w_{i-1}^{-1}$. The element $w_{0 i}$ is fixed for a particular group $G$ and is the unique $W_{J_{i+1}}$-reduced element of $W_{J_{i}}$ such that $\lambda_{i} w_{0 i}^{-1}=\lambda_{i} w_{0 J_{i}}$. Iterating the result in the previous subsection, we can now refine the Bruhat decomposition to

$$
\bigcup_{w_{1}, \ldots, w_{k}} U_{w_{01}} U_{w_{02}} \cdots U_{w_{0 k}} H \dot{w}_{k} \cdots \dot{w}_{2} \dot{w}_{1}\left(U_{w_{k}}\right)^{\dot{w}_{k-1} \cdots \dot{w}_{1}} \cdots\left(U_{w_{2}}\right)^{\dot{w}_{1}} U_{w_{1}},
$$

where $w_{j}$ ranges over the $W^{(j+1)}$-reduced elements of $W^{(j)}$.

7.5. Row and column reduction. Putting together the ideas of the previous sections, we get Algorithm [ (see Section 8.1 for the significance of the asterisks). We assume that $\lambda_{i}, J_{i}$ and $w_{0 i}$ have already been computed using the techniques of Section 7.4. Note that the loop has an invariant $\rho(u) A \rho\left(u^{\prime}\right)$, which is always equal to the original value of $A$. At the end of this algorithm, $A$ is the image of a torus element. 


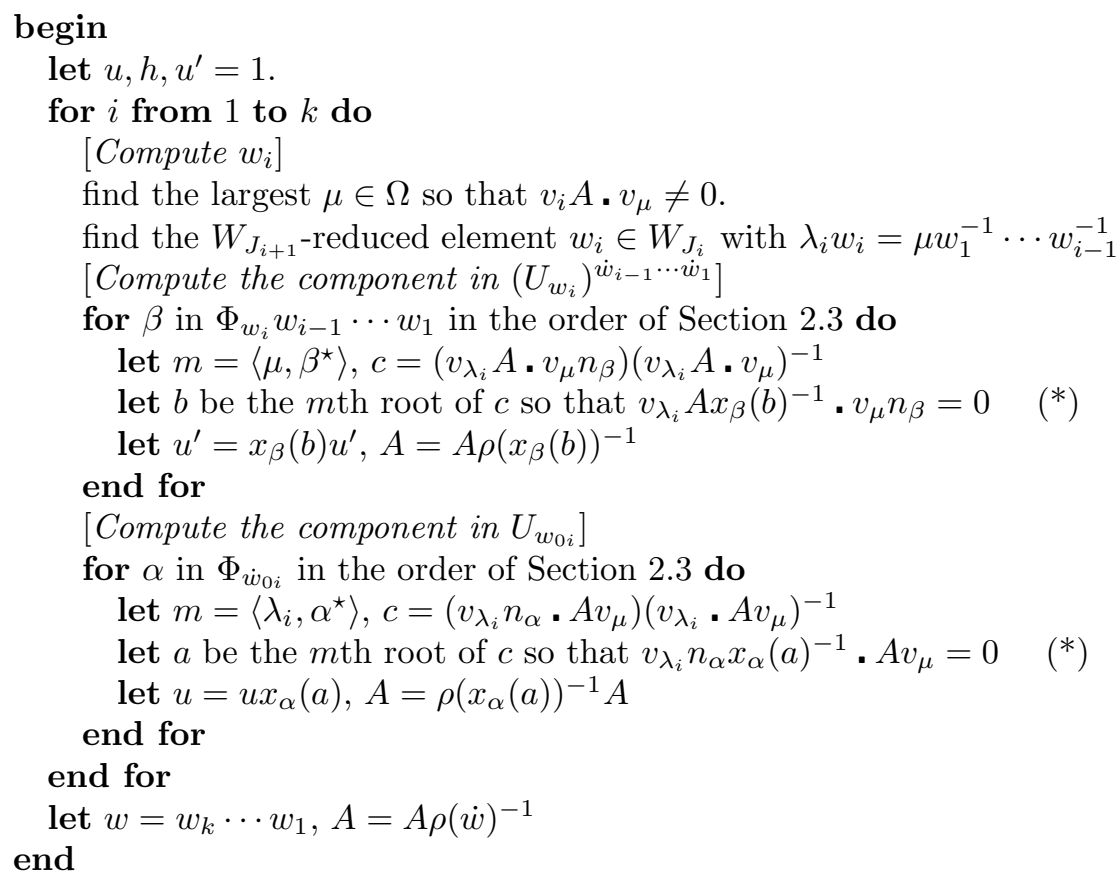

Algorithm 6. Generalised row and column reduction

7.6. Torus elements. We now suppose we have an element $A \in \rho(H)$ where $H$ is the torus of our group of Lie type $G$, and we wish to find $h \in H$ so that $\rho(h)=A$. Note that $\rho$ need not be faithful on $H$, so $h$ may not be uniquely determined. Let $\Gamma$ be the lattice generated by the weights of $V$ and choose a basis $\gamma_{1}, \ldots, \gamma_{t}$ for $\Gamma$. Then the preimage of $A$ in $\Gamma \otimes \mathbb{F}^{\times}$is computed as $\prod_{i=1}^{t} \gamma_{i} \otimes t_{i}$ where $v A=t_{i} v$ for $v \in V_{\gamma_{i}}$. This can be converted to the form $\prod_{i=1}^{t} f_{i} \otimes h_{i}$ using the methods of Section 5.2 .

\section{Applications and example}

The algorithms described in this paper have been implemented in Magma [2]. They are practical for computations in groups of Lie type up to (and sometimes beyond) rank 10. In particular, they are practical for all the exceptional groups. The timings for multiplication in the Steinberg presentation are highly dependent on the efficiency of the field operations. However, it is possible to do computations in $E_{8}$ even for quite difficult fields, such as fields of rational functions. Rational function fields are particularly useful, since a computation with indeterminants allows us to derive general formulas that apply over any field.

Most of the applications of our algorithms are based on the ability to convert in both directions between the Steinberg presentation and a representation. This allows us to work in whichever of the two is most convenient for the problem at hand: in general the Steinberg presentation has the advantage of making the underlying Lie theory explicit, while a representation allows us to use the power of linear algebra. For example, conjugacy classes are more easily computed in the 
presentation, while the Jordan decomposition and lattice subgroups can only be computed in a representation.

8.1. Application to matrix group recognition. One of the more difficult problems in matrix group recognition is finding a membership test for a known almost simple group. Our algorithm provides such a test for natural characteristic representations of finite groups of Lie type. We obtain a membership test for $\rho(G)$ by running our algorithm for an arbitrary $A \in \mathrm{GL}(V): A$ is not a member of $\rho(G)$ if the algorithm fails or the value of $A$ at the end is not the identity matrix. Algorithm [6 can fail in three places, which are marked by $\left(^{*}\right)$. The torus element algorithm of Section 7.6 can also fail.

In this and other applications, it is of interest to determine whether our algorithm runs in polynomial time. When $\mathbb{F}$ is a finite field of order $q$, it is straightforward to show that most of the algorithm runs in polynomial time in $\log (q)$ and the coefficients of $\lambda$. For example, the length $k$ of our base is bounded by the reductive rank of $G$ and radicals over finite fields can be computed in polynomial time by [12. The main sticking point is the computation of discrete logarithms in Section 7.1 This can be avoided, however, since the powers involved are bounded by $m$, and by Section 7.3 the values of $m$ which appear are polynomial in the coefficients of $\lambda$. We now have the following result.

Theorem 8.1. Let $G=G_{\mathcal{R}}\left(\mathbb{F}_{q}\right)$ be a finite group of Lie type and let $\rho: G \rightarrow$ $\mathrm{GL}(V)$ be a quotient of a highest weight representation with known highest weight $\lambda$. Suppose $A \in \mathrm{GL}(V)$. We can decide whether $A$ is in $\rho(G)$ and, if it is, find a preimage in polynomial time in $\log (q)$ and the coefficients of $\lambda$.

Note that the condition that the highest weight be known is required, since computing the highest weight requires discrete logarithms. In fact, if $G$ is a onedimensional torus with representation $V$, then computing the weight of $V$ is precisely the discrete logarithm problem.

As an immediate corollary of Theorem 8.1 , we get a polynomial time algorithm for multiplying elements in $G$ : simply convert the elements to matrices in the natural representation (i.e., the smallest degree highest weight representation), multiply the matrices, then convert back. In the case of the natural representation, the running time of our algorithm is $O\left(n^{3}\right)$, just like the LUP algorithm for general linear groups.

8.2. Example: $\mathrm{GL}_{n+1}(\mathbb{F})$. We now describe how this algorithm works in the case of the standard representation of the general linear group. Take $X=\mathbb{Z}^{n+1}$ with standard basis vectors $e_{i}$ and $Y=\mathbb{Z}^{n+1}$ with standard basis vectors $f_{i}$. The roots and coroots are of the form $\alpha_{i j}=e_{i}-e_{j}$ and $\alpha_{i j}^{\star}=f_{i}-f_{j}$ for $1 \leq i, j \leq n+1$ with $i \neq j$. A (co)root is positive when $i>j$ and simple when $i=j+1$.

The standard representation $\rho: G \rightarrow \mathrm{GL}_{n+1}(\mathbb{F})$ takes $x_{\alpha_{i j}}(a)$ to the matrix equal to the identity except for the $(i, j)$-entry which is equal to $a$, and it takes $f_{i} \otimes t$ to the matrix equal to the identity except for the $(i, i)$-entry which is equal to $t$. The Weyl group of $G$ is the symmetric group on $n+1$ letters with reflections $s_{\alpha_{i j}}=(i, j)$. Here $\rho\left(g^{\delta}\right)$ is the usual transpose of $\rho(g)$.

Algorithm 5 gives $\lambda_{i}=e_{i}$ and $J_{i}=\{i, i+1, \ldots, n+1\}$. Since we can take $v_{\lambda_{i}}=b_{i}$, these weights correspond to the rows and columns of the input matrix $A$. 
We can compute

$$
w_{0 i}=s_{n} \ldots s_{i+1} s_{i}=(i, i+1, \ldots, n+1)
$$

and

$$
\Phi_{w_{0 i}}=\left\{\alpha_{i+1, i}, \ldots, \alpha_{n+1, i}\right\} .
$$

In order to compute $w_{i}$, we need to know the image

$$
\lambda_{i} w_{i}=\mu w_{1}^{-1} \cdots w_{i-1}^{-1}
$$

where $\mu=e_{j}$ with $j$ the largest nonzero entry in the $i$ th row of $A$. Now

$$
w_{i}=s_{i} s_{i+1} \ldots s_{i w_{i}-1}=\left(i w_{i}, \ldots, i+1, i\right)
$$

and

$$
\Phi_{w_{i}}=\left\{\alpha_{i w_{i}, i}, \alpha_{i w_{i}, i+1}, \ldots, \alpha_{i w_{i}, i w_{i}-1}\right\}
$$

and so

$$
\Phi_{w_{i}} w_{i-1} \cdots w_{1}=\left\{\alpha_{i w, j} \mid j=l w_{i-1} \cdots w_{1} \text { for } l=i, i+1, \ldots, i w_{i}-1\right\} .
$$

Furthermore the constants $m$ that appear in Algorithm 6 are all equal to 1 . It is now easily seen that computing the $U_{w_{0 i}}$ component of (32) is just clearing the $\left(i w_{i-1} \cdots w_{1}\right)$ th column of the matrix with row operations, and computing the $\left(U_{w_{i}}\right)^{\dot{w}_{i-1} \cdots \dot{w}_{1}}$ component is just clearing the $i$ th row of the matrix with column operations (the values of $j$ that appear in (33) give exactly the entries in the row that have not been cleared by a previous column operation).

In order to relate this algorithm to the well-known LUP algorithm, we note that $\rho$ can be chosen so that $\rho(B)$ consists of lower triangular matrices. Suppose that

$g \in G$ decomposes as $u h \dot{w} u^{\prime}$ for $u \in U, h \in H, w \in W$ and $u^{\prime} \in U_{w}$. Then $L=\rho(u h)$ is lower triangular, $U=\rho\left(\dot{w} u^{\prime} \dot{w}^{-1}\right)$ is upper triangular, and $P=\rho(\dot{w})$ is a permutation matrix (up to signs), giving the usual LUP decomposition.

\section{REFERENCES}

[1] J. L. Alperin and Rowen B. Bell, Groups and representations, Springer-Verlag, New York, 1995. MR 96m:20001

[2] W. W. Bosma and J. J. Cannon, Handbook of magma functions, School of Mathematics and Statistics, University of Sydney, Sydney, 1997.

[3] L. Babai, A. J. Goodman, W. M. Kantor, E. M. Luks, and P. P. Pálfy, Short presentations for finite groups, J. Algebra 194 (1997), no. 1, 79-112. MR 98h:20044

[4] Nicolas Bourbaki, Éléments de mathématique, Masson, Paris, 1981, Groupes et algèbres de Lie. Chapitres 4, 5 et 6. [Lie groups and Lie algebras. Chapters 4, 5 and 6]. MR 83g:17001]

[5] G. Butler, Fundamental algorithms for permutation groups, Springer-Verlag, Berlin, 1991. MR 94d:68049

[6] Roger W. Carter, Simple groups of Lie type, John Wiley \& Sons, London-New York-Sydney, 1972, Pure and Applied Mathematics, Vol. 28. MR 53:10946

[7] Roger W. Carter, Finite groups of Lie type, John Wiley \& Sons Ltd., Chichester, 1993, Conjugacy classes and complex characters, Reprint of the 1985 original, A Wiley-Interscience Publication. MR 94k:20020

[8] C. Chevalley, Sur certains groupes simple, Tôhoku Math. J. (2) 7 (1955), 14-66. MR 17:457c

[9] M. Demazure, Données radicielles, Schémas en Groupes (Sém. Géométrie Algébrique, Inst. Hautes Études Sci., 1964), Fasc. 6, Exposé 21, Inst. Hautes Études Sci., Paris, 1965. MR 34:7524

[10] Willem A. de Graaf, Lie algebras: theory and algorithms, North-Holland Publishing Co., Amsterdam, 2000. MR 2001j:17011 
[11] W. A. de Graaf, Constructing representations of split semisimple Lie algebras, J. Pure Appl. Algebra 164 (2001), no. 1-2, 87-107, Effective methods in algebraic geometry (Bath, 2000). MR 2002h: 17007

[12] P. Flajolet, X. Gourdon, and D. Panario, The complete analysis of a polynomial factorization algorithm over finite fields, J. Algorithms 40 (2001), no. 1, 37-81. MR 2002f:68193

[13] I. B. Frenkel and V. G. Kac, Basic representations of affine Lie algebras and dual resonance models, Invent. Math. 62 (1980/81), no. 1, 23-66. MR 84f:17004

[14] The GAP Group, Aachen, St Andrews, GAP - Groups, Algorithms, and Programming, Version 4.1, 1999 (http://www.gap-system.org).

[15] Meinolf Geck, Gerhard Hiss, Frank Lübeck, Gunter Malle, and Götz Pfeiffer, CHEVIE-a system for computing and processing generic character tables, Appl. Algebra Engrg. Comm. Comput. 7 (1996), no. 3, 175-210, Computational methods in Lie theory (Essen, 1994). MR 99m:20017

[16] Peter B. Gilkey and Gary M. Seitz, Some representations of exceptional Lie algebras, Geom. Dedicata 25 (1988), no. 1-3, 407-416, Geometries and groups (Noordwijkerhout, 1986). MR 89h:20056

[17] Sergei Haller, Unipot-a system for computing with elements of unipotent subgroups of Chevalley groups, version 1.1, Tech. report, Justus-Liebig Universität, Germany, July 2000, http://www.gap-system.org/Info4/deposit.html\#pkg.

[18] R. B. Howlett, L. J. Rylands, and D. E. Taylor, Matrix generators for exceptional groups of Lie type, J. Symbolic Comput. 31 (2001), no. 4, 429-445. MR 2002c:20078

[19] Alexander Hulpke and Ákos Seress, Short presentations for three-dimensional unitary groups, J. Algebra 245 (2001), no. 2, 719-729. MR 2002m:20079

[20] William M. Kantor and Ákos Seress, Black box classical groups, Mem. Amer. Math. Soc. 149 (2001), no. 708, viii+168. MR 2001m:68066

[21] Charles R. Leedham-Green, The computational matrix group project, Groups and computation, III (Columbus, OH, 1999), de Gruyter, Berlin, 2001, pp. 229-247. MR 2002d:20084

[22] C. R. Leedham-Green and L. H. Soicher, Collection from the left and other strategies, J. Symbolic Comput. 9 (1990), no. 5-6, 665-675, Computational group theory, Part 1. MR 92b:20021

[23] Martin W. Liebeck and Jan Saxl, On the orders of maximal subgroups of the finite exceptional groups of Lie type, Proc. London Math. Soc. (3) 55 (1987), no. 2, 299-330. MR 89b:20068

[24] R. J. Riebeek, Computations in association schemes, Ph.D. thesis, Eindhoven University of Technology, 1998.

[25] L. J. Rylands, A formula for signs of structure constants, Unpublished, 2001.

[26] Charles C. Sims, Computation with finitely presented groups, Cambridge University Press, Cambridge, 1994. MR 95f:20053

[27] T. A. Springer, Linear algebraic groups, second ed., Birkhäuser Boston Inc., Boston, MA, 1998. MR 99h:20075

[28] Robert Steinberg, Générateurs, relations et revêtements de groupes algébriques, Colloq. Théorie des Groupes Algébriques (Bruxelles, 1962), Librairie Universitaire, Louvain, 1962, pp. 113-127. MR 27:3638

[29] Robert Steinberg, Representations of algebraic groups, Nagoya Math. J. 22 (1963), 33-56. MR 27:5870

[30] R. Steinberg, Lectures on Chevalley groups, Tech. report, Yale University, 1968. MR 57:6215

[31] M. A. A. van Leeuwen, A. M. Cohen, and B. Lisser, LiE manual, CWI/CAN, Amsterdam, 1992, Manual for the software package LiE for Lie group theoretical computations. http://young.sp2mi.univ-poitiers.fr/ marc/LiE/.

Department of Mathematics and Computer Science, Eindhoven University of Technology, PO Box 513, 5600 MB Eindhoven, The Netherlands

E-mail address: A.M.Cohen@tue.nl

Department of Mathematics and Statistics, University of Sydney, Sydney, New South Wales 2006, Australia

E-mail address: murray@maths.usyd.edu.au

Department of Mathematics and Statistics, University of Sydney, Sydney, New South Wales 2006, Australia

E-mail address: D.Taylor@maths.usyd.edu.au 